\title{
Studies with 2-arylhydrazono-3-oxopropanals: routes for the synthesis of pyridazine-3,4-dicarboxylate and 3,5-diaroyl pyrazoles
}

\author{
Mariam A. Al-Shiekh, ${ }^{\text {a }}$ Hanady Y. Medrassi, ${ }^{a}$ Mohamed H. Elnagdi, ${ }^{b}$ and Ebtisam A. \\ Hafez $^{\mathfrak{c}_{*}}$ \\ ${ }^{a}$ Department of Chemistry, Girls College of Education, Jeddah, P. O. Box 138016 \\ Jeddah 21323, Kingdom of Saudi Arabia \\ ${ }^{b}$ Department of Chemistry; Faculty of Science; University of Kuwait, P. O. Box 5969 Safat, \\ 13060 Kuwait \\ ${ }^{c}$ Department of Chemistry; Faculty of Science; Cairo University, Giza-12613 Egypt \\ E-mail: ebtisamhafez@,hotmail.com
}

\begin{abstract}
The title compounds 3a-j were synthesized via coupling of enaminones 2a-d with aromatic diazonium salts. The reaction of $\mathbf{3 b - f}, \mathbf{h}-\mathbf{j}$ with dimethyl acetylenedicarboxylate and triphenylphosphine afforded dimethyl 2-aryl-6-aroyl-2,3-dihydropyridazine-3,4-dicarboxylates $\mathbf{7 b - f , h - j}$. . The reaction of $\mathbf{3 b}, \mathbf{d}, \mathbf{f}, \mathbf{g}$ with phenacyl bromide afforded 3-aroyl-5-benzoylpyrazoles 9b,d,f,g., while compound 3i condensed with benzoylacetonitrile to yield pyridazin-6-imine 11.Reaction of $\mathbf{3 c - e , h , j}$ with $p$-toluidine yielded the enamineazo $\mathbf{1 2} \mathbf{c}-\mathbf{e}, \mathbf{h}, \mathbf{j}$. The structures of $\mathbf{7 b}, \mathbf{d}, \mathbf{i}$ and $\mathbf{9 b}$ were confirmed by X-ray crystal structure determination.
\end{abstract}

Keywords: 2-Arylhydrazonopropanals, enaminones, pyridazine-3,4-dicarboxylate, 3,5diaroylpyrazoles

\section{Introduction}

The coupling reaction of functionally substituted enamines of type $\mathbf{2}$ with aromatic diazonium salts ${ }^{1}$ has opened the route for the synthesis of 2-arylhydrazonals 3 . It was shown that derivatives of 3 are useful starting materials for a variety of heterocycles. ${ }^{2-6}$ Therefore, in conjunction with this work, we report this study aimed at establishing, with certainty the previous conclusions. Our results were confirmed via X-ray crystal determinations and extended previously reported findings ${ }^{6}$ in order to see if this constitutes a new general route for the preparation of dialkylpyridazine dicarboxylates as well as 3,5-diaroylpyrazoles. 


\section{Results and Discussion}

Enaminones $\mathbf{2}$ were previously synthesized by condensing heteroaryl methyl ketones $\mathbf{1 a , b}$ and arylmethyl ketones 1c-e with dimethylformamide dimethylacetal DMFDMA in xylene following some literature procedures. ${ }^{1,7}$ However, only moderate yields of the desired products 2a-e were obtained. Consequently we have modified this synthetic approach and condensed these ketones with DMFDMA in absence of solvent. In this case, the required enaminones were obtained almost in better yield, and this method is more economic. Yields and reaction times for both methods are reported in Table1.

Table 1. Comparison between reaction time and yields obtained from conventional heating in solvent and without solvent

\begin{tabular}{lllll}
\hline $\begin{array}{l}\text { Sample } \\
\text { NO. }\end{array}$ & \multicolumn{2}{l}{$\Delta$ in xylene } & \multicolumn{2}{l}{$\Delta$ in excess DMFDMA } \\
\cline { 2 - 5 } & Time(min.) & Yield $\%$ & Time(min.) & Yield \% \\
\hline $\boldsymbol{2 a}$ & 450 & 70 & 480 & 80 \\
$\boldsymbol{2 \boldsymbol { b }}$ & 450 & 60 & 480 & 77 \\
$\boldsymbol{2 c}$ & 450 & 60 & 480 & 85 \\
$\boldsymbol{2 \boldsymbol { d }}$ & 450 & 70 & 480 & 89 \\
$\boldsymbol{2} \boldsymbol{e}$ & 450 & 61 & 480 & 88 \\
\hline
\end{tabular}

Coupling enaminones $\mathbf{2}$ with aromatic diazonium salts afforded the products $\mathbf{3 a} \mathbf{a} \mathbf{j}$ which were previously shown to exist, in the solid state, in the anti form according to the X-ray crystal structure determination of $\mathbf{3 a}, \mathbf{h}$ published recently by our group. ${ }^{8}$ This agrees with a recent observation $^{9}$ that in the 2-arylhydrazonoketones stereoelectronic factors overweigh any possible lock of conformation that may occur due to hydrogen bonding-(Scheme 1). We observed in previous work ${ }^{6}$ that other derivatives of 3 reacted with dimethyl acetylene dicarboxylate DMAD in the presence of triphenylphosphine to yield pyridazine-3,4-dicarboxylates. In the present work 3b-f,h-j reacted with DMAD yielding $\mathbf{7 b} \mathbf{b}-\mathbf{f}, \mathbf{h}-\mathbf{j}$ (Scheme 1). Previously the mechanism for the formation of the end products ${ }^{6}$ that was suggested could not be supported by evidence and the proposed structure did not seem completely convincing. By obtaining X-ray crystal structures for $\mathbf{7 b}, \mathbf{d}, \mathbf{i}$ we could confirm our conclusions (cf. Figures 1-4). ${ }^{10}$

Recently, ${ }^{11}$ it has been reported the first synthesis of 5-acetyl-3-aroylpyrazoles by mixing derivatives of $\mathbf{3}$ with chloroacetone. Now we have found that $\mathbf{3 b}, \mathbf{d , f}, \mathbf{g}$ also react to yield colored products 9b,d,f,g, of molecular formulae corresponding to their condensation with phenacyl bromide via hydrogen bromide and water elimination thus, the alternative furan structure 10 could be ruled out. X-ray crystal structure determination confirmed that the reaction products are in fact 9 (Scheme 1, Fig. 4) formed most likely via intermediacy of $\mathbf{8}$. Compound $\mathbf{3 g}$ condensed also with benzoylacetonitrile to yield pyridazin-6-imine 11, based on spectral data. 
2-Arylhydrazono-3-propanals $\mathbf{3 c - e , h , j}$ reacted with $p$-toluidine to yield products of condensation via water elimination. Two tautomeric forms are possible for these condensation products enamineazo 12 or iminohydrazone isomer 13. ${ }^{1} \mathrm{H}$ NMR data clearly revealed that this condensation has involved the formyl carbon as the formyl proton has disappeared and also ${ }^{1} \mathrm{H}$

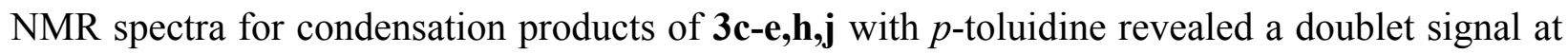
$\delta=8.6 \mathrm{ppm}(\mathrm{J}=7.2 \mathrm{~Hz})$ for $\mathrm{HCNH}$ proton and another signal at $\delta=15.4 \mathrm{ppm}$ for $\mathrm{NH}$ proton. The fact that the proton attached to the carbon atom linked to the amine nitrogen appeared as a doublet allowed us to suggest the enaminoazo structures $\mathbf{1 2}$ for these products(Scheme 1).

\section{Conclusions}

In conclusion, 2-arylhydrazonopropanals can serve as starting materials for the synthesis of a variety of heteroaromatic compounds with an interesting substitution pattern and the efficient synthesis of enaminones achieved in this work makes also these compounds economically acceptable as starting materials. Moreover, previously assigned studies could be unambiguously established.

\section{Crystallographic Analysis}

The crystals were mounted on a glass fiber. All measurements were performed on an ENRAF NONIUNS FR 590. The data were collected at temperature of $20 \pm 1{ }^{\circ} \mathrm{C}$ using the $\omega$ scanning technique to a maximum of a 20 of $27.12^{\circ}$. The structure was solved by the direct method using SIR $92^{12}$. Non-hydrogen atoms were refined anisotropically by full matrix least squares. Hydrogen atoms were located geometrically and were refined isotropically. ${ }^{13}$ 


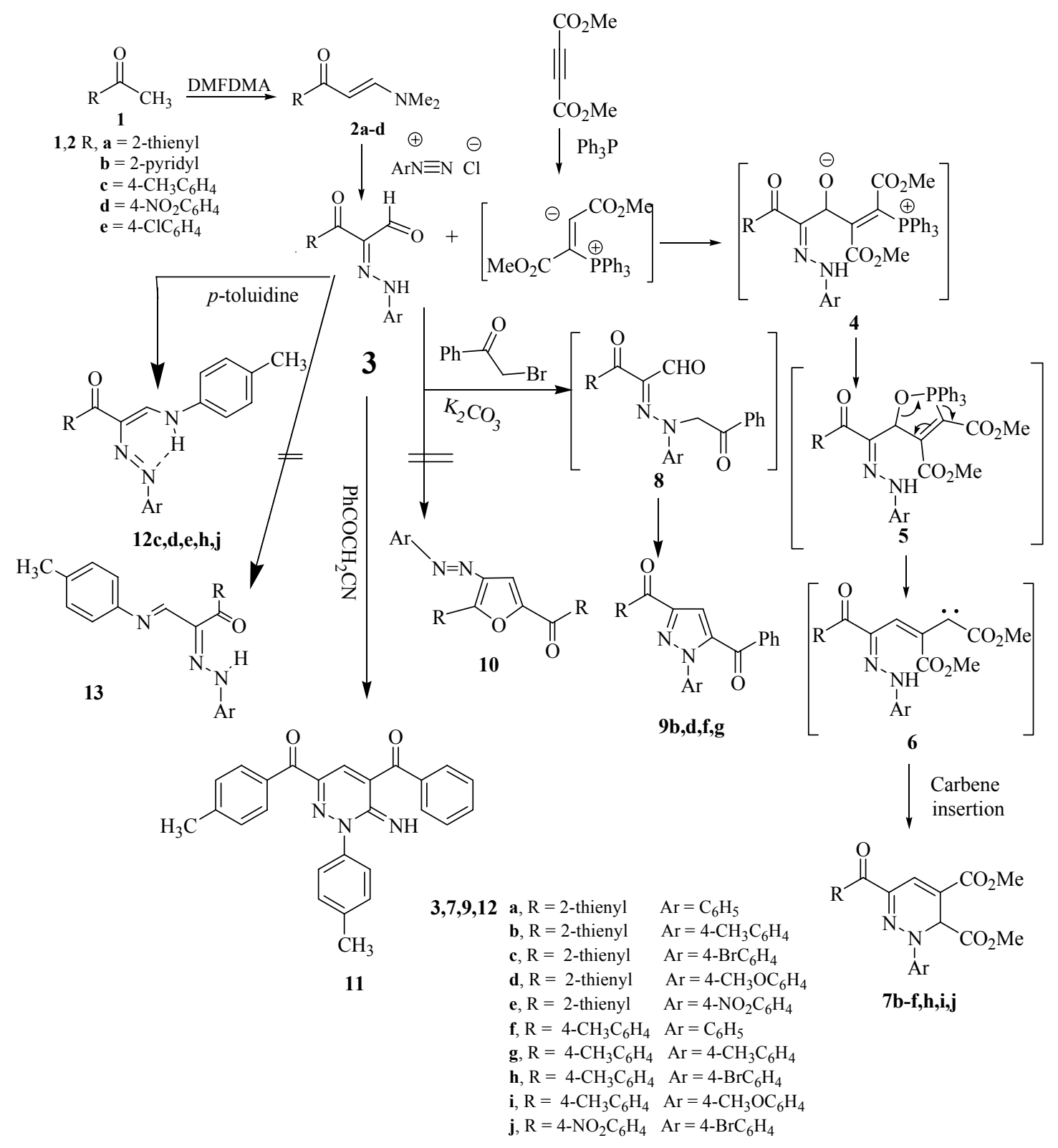

\section{Scheme 1}




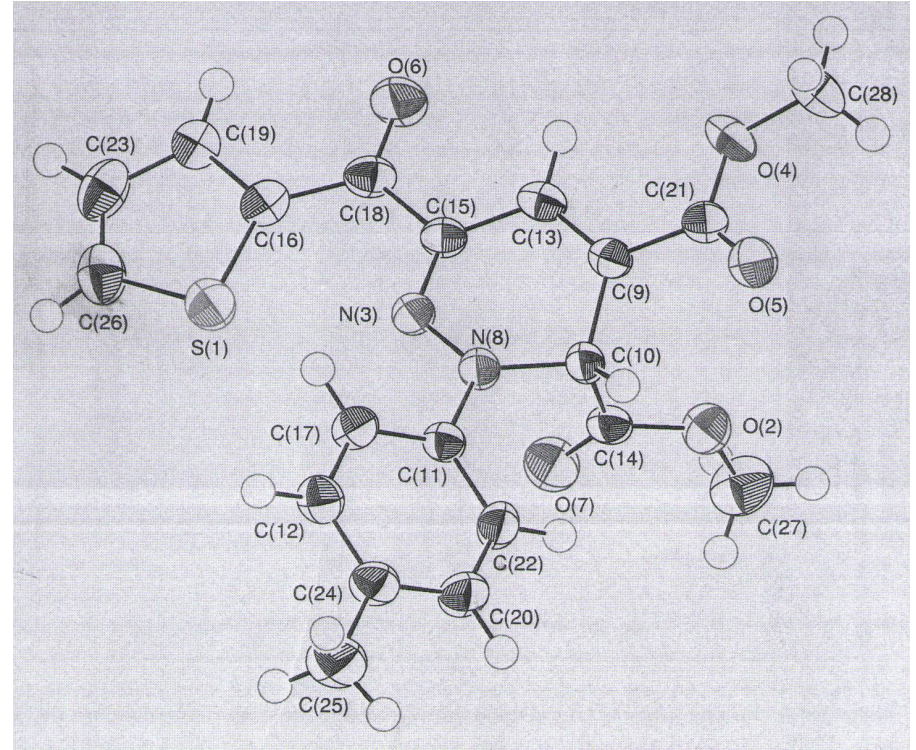

Figure 1. Thermal ellipsoid plots of the X-ray structure of $7 \mathbf{b}$ ( $50 \%$ probability).
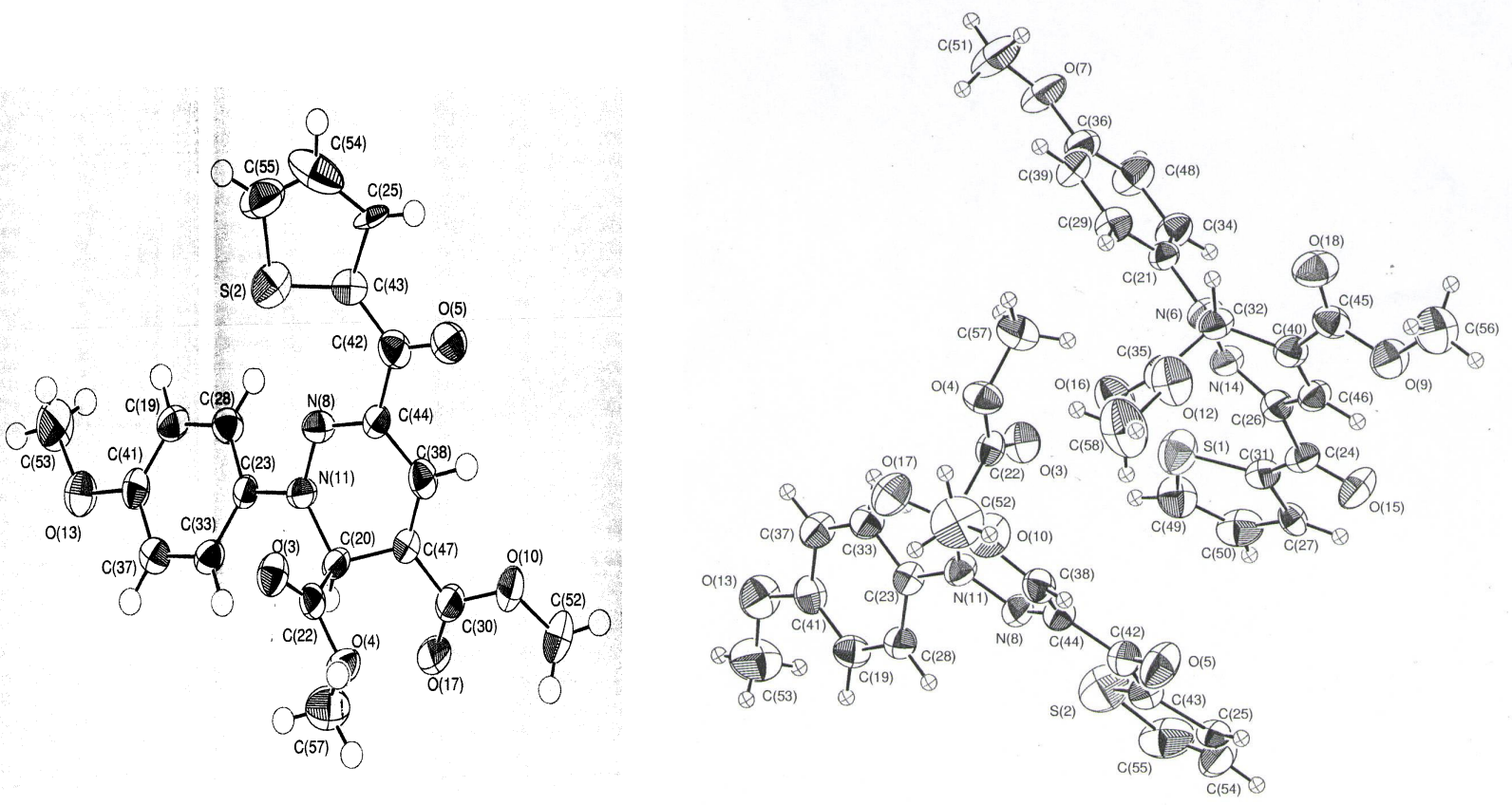

Figure 2. Thermal ellipsoid plots of the X-ray structure of $7 \mathbf{d}$ ( $50 \%$ probability). 


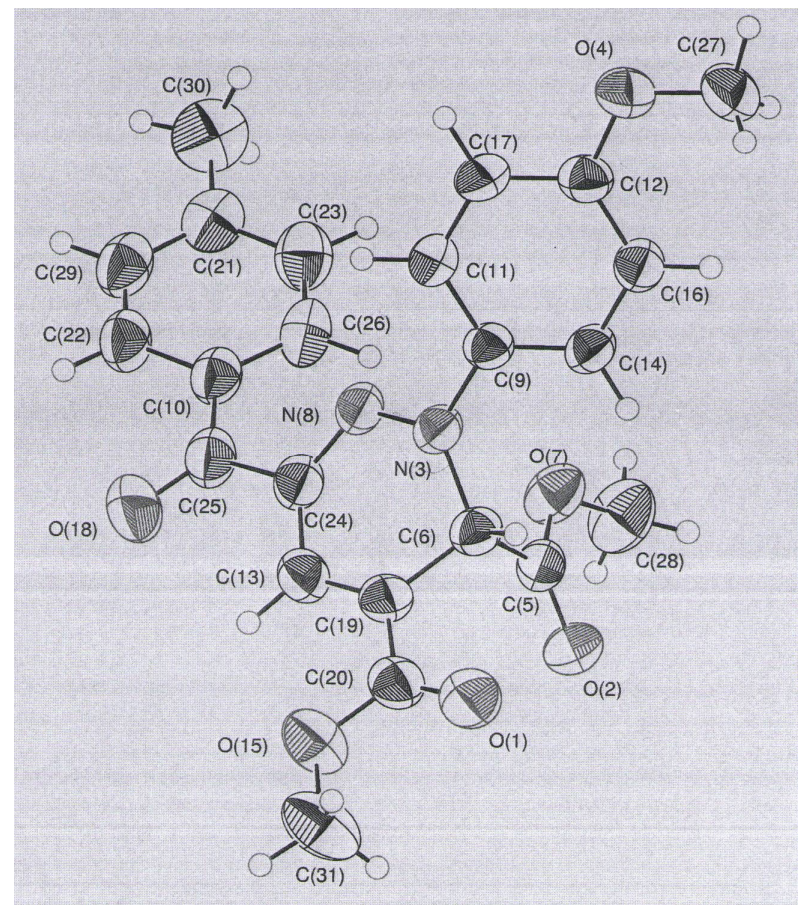

Figure 3. Thermal ellipsoid plots of the X-ray structure of $7 \mathbf{i}$ (50\% probability.

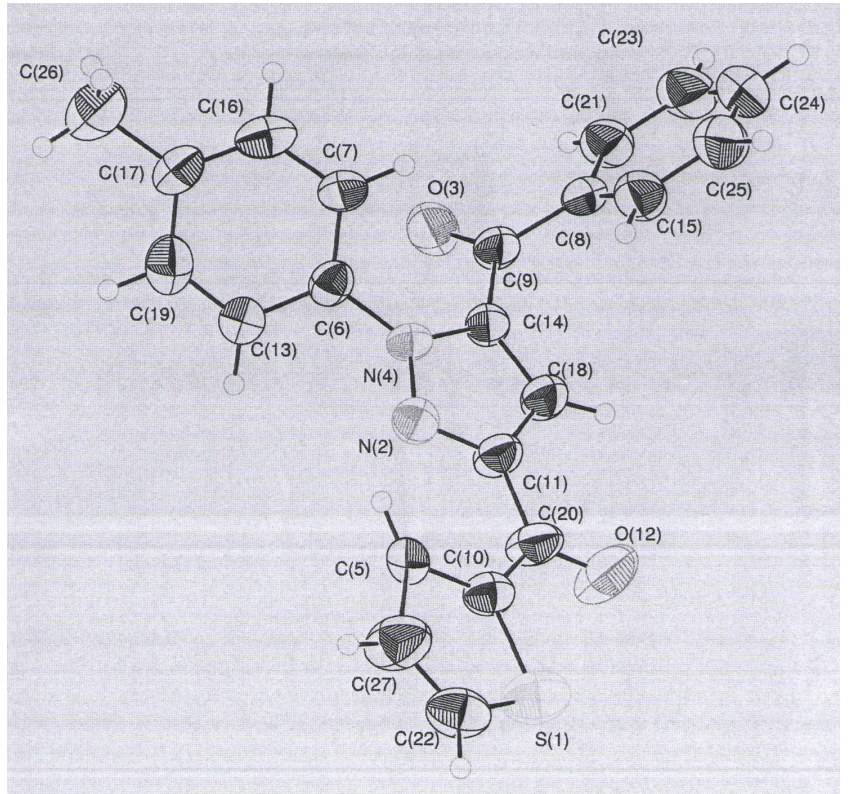

Figure 4. Thermal ellipsoid plots of the X-ray structure of $9 \mathbf{b}$ (50\% probability). 
Table 2. Crystal data and structure refinement for compounds $\mathbf{7 b , d , i}$ and $\mathbf{9 b}$

\begin{tabular}{|c|c|c|c|c|}
\hline & $\begin{array}{l}7 \mathrm{~b} \\
\text { CCDC } 617787\end{array}$ & $\begin{array}{l}7 d \\
\text { CCDC } 617786 \\
\end{array}$ & $\begin{array}{l}7 i \\
\text { CCDC } 617788 \\
\end{array}$ & $\begin{array}{l}\text { 9b } \\
\text { CCDC } 617777\end{array}$ \\
\hline Empirical Formula & $\mathrm{C}_{20} \mathrm{H}_{18} \mathrm{~N}_{2} \mathrm{O}_{5} \mathrm{~S}$ & $\mathrm{C}_{20} \mathrm{H}_{18} \mathrm{~N}_{2} \mathrm{O}_{6} \mathrm{~S}$ & $\mathrm{C}_{23} \mathrm{H}_{22} \mathrm{~N}_{2} \mathrm{O}_{6}$ & $\mathrm{C}_{22} \mathrm{H}_{16} \mathrm{~N}_{2} \mathrm{O}_{2} \mathrm{~S}$ \\
\hline Formula weight & 398.437 & 414.436 & 453.407 & 372.446 \\
\hline Crystal System & Triclinic & Triclinic & Monoclinic & Triclinic \\
\hline Space group & $\mathrm{P}-1$ & $\mathrm{P}-1$ & $\mathrm{P} 21 / \mathrm{c}$ & $\mathrm{P}-1$ \\
\hline $\begin{array}{l}\text { Unit cell parameters } \\
\mathrm{a}[\AA] \\
\mathrm{b}[\AA] \\
\mathrm{c}[\AA] \\
\alpha^{\mathrm{o}} \\
\beta^{\mathrm{o}} \\
\gamma^{\mathrm{o}}\end{array}$ & $\begin{array}{l}7.9742(2) \\
9.5600(3) \\
13.4031(4) \\
79.5153(12) \\
73.4154(13) \\
89.1275(11) \\
\end{array}$ & $\begin{array}{l}9.7889(2) \\
13.3834(3) \\
16.8502(5) \\
69.6369(8) \\
76.0099(9) \\
81.6577(8) \\
\end{array}$ & $\begin{array}{l}8.0662(2) \\
21.1130(4) \\
12.3971(3) \\
90.00 \\
95.7775(9) \\
90.00 \\
\end{array}$ & $\begin{array}{l}8.5993(3) \\
10.9802(3) \\
11.1537(5) \\
116.9690(14) \\
91.8325(14) \\
95.353(2) \\
\end{array}$ \\
\hline $\begin{array}{l}\text { Unit cell volume } \\
\mathrm{Z}\end{array}$ & $\begin{array}{l}962.18(5) \\
2\end{array}$ & $\begin{array}{l}2003.66(9) \\
4\end{array}$ & $\begin{array}{l}2100.52(8) \\
4\end{array}$ & $\begin{array}{l}931.25(6) \\
2\end{array}$ \\
\hline Temperature (K) & 298 & 298 & 298 & 298 \\
\hline Radiation type & MoKá & MoKá & MoKá & MoKá \\
\hline $\mathrm{F}(000)$ & 416 & 864 & 944 & 388 \\
\hline $\begin{array}{l}\text { Absorption coefficient } \\
\left(\mathrm{mm}^{-1}\right)\end{array}$ & 0.20 & 0.20 & 0.11 & 0.19 \\
\hline Parameters & 253 & 523 & 280 & 239 \\
\hline $\mathrm{R}$ factor & 0.050 & 0.061 & 0.048 & 0.089 \\
\hline
\end{tabular}


Table 3. Selected bond length $[\AA]$ and angles $\left[{ }^{\circ}\right]$ for compounds $\mathbf{7 b}, \mathbf{d}, \mathbf{i}$ and $\mathbf{9 b}$

\begin{tabular}{|c|c|c|c|c|c|c|c|c|c|c|c|}
\hline \multicolumn{3}{|c|}{ Bond lengths $\mathbf{7 b}$} & \multicolumn{3}{|c|}{ Bond lengths $\mathbf{7 d}$} & \multicolumn{3}{|c|}{ Bond lengths $7 \mathbf{i}$} & \multicolumn{3}{|c|}{ Bond lengths $\mathbf{9 b}$} \\
\hline N3 N8 & \multicolumn{2}{|c|}{$1.332(3)$} & N8 N11 & \multicolumn{2}{|c|}{$1.335(3)$} & \multicolumn{2}{|c|}{ N3 N8 } & $1.328(2)$ & $\mathrm{N} 2 \mathrm{~N} 4$ & \multicolumn{2}{|l|}{$1.337(5)$} \\
\hline N3 C15 & \multicolumn{2}{|c|}{$1.313(3)$} & N8 C44 & \multicolumn{2}{|c|}{$1.319(3)$} & \multicolumn{2}{|c|}{ N3 C6 } & $1.468(2)$ & $\mathrm{N} 2 \mathrm{C} 11$ & \multicolumn{2}{|l|}{$1.335(6)$} \\
\hline $\mathrm{N} 8 \mathrm{C} 10$ & \multicolumn{2}{|c|}{$1.474(3)$} & C44 C38 & \multicolumn{2}{|c|}{$1.426(4)$} & \multicolumn{2}{|c|}{ C6 C19 } & $1.503(3)$ & $\mathrm{N} 4 \mathrm{C} 14$ & \multicolumn{2}{|l|}{$1.366(6)$} \\
\hline $\mathrm{C} 10 \mathrm{C} 14$ & \multicolumn{2}{|c|}{$1.504(3)$} & $\mathrm{N} 11 \mathrm{C} 23$ & \multicolumn{2}{|c|}{$1.431(3)$} & \multicolumn{2}{|c|}{ N3 C9 } & $1.426(2)$ & $\mathrm{C} 6 \mathrm{C} 7$ & \multicolumn{2}{|l|}{$1.375(6)$} \\
\hline $\mathrm{C} 10 \mathrm{H} 10$ & \multicolumn{2}{|c|}{$0.960(2)$} & $\mathrm{C} 20 \mathrm{C} 47$ & \multicolumn{2}{|c|}{$1.518(3)$} & \multicolumn{2}{|c|}{$\mathrm{C} 13 \mathrm{C} 24$} & $1.432(3)$ & $\mathrm{C} 9 \mathrm{C} 14$ & \multicolumn{2}{|l|}{$1.485(6)$} \\
\hline $\mathrm{C} 13 \mathrm{C} 15$ & \multicolumn{2}{|c|}{$1.434(3)$} & $\mathrm{N} 11 \mathrm{C} 20$ & \multicolumn{2}{|c|}{$1.464(3)$} & \multicolumn{2}{|c|}{$\mathrm{C} 9 \mathrm{C} 14$} & $1.378(2)$ & $\mathrm{C} 11 \mathrm{C} 18$ & \multicolumn{2}{|l|}{$1.394(7)$} \\
\hline $\mathrm{C} 13 \mathrm{C} 9$ & \multicolumn{2}{|c|}{$1.343(3)$} & $\mathrm{C} 38 \mathrm{C} 47$ & \multicolumn{2}{|c|}{$1.336(3)$} & $\mathrm{C} 14$ & 16 & $1.395(3)$ & $\mathrm{C} 14 \mathrm{C} 18$ & $1.363(6)$ & \\
\hline Bond angle & $7 \mathrm{~b}$ & & Bond an & les $\mathbf{7 d}$ & & & Bon & angles $\mathbf{7 i}$ & & Bond angles 9 & \\
\hline N8 N3 C15 & & $118.4(2)$ & N8 N11 & & & $.6(2)$ & N8 & $3 \mathrm{C} 9$ & $116.13(14)$ & N4 N2 C11 & 105.1(4) \\
\hline $\mathrm{C} 9 \mathrm{C} 10 \mathrm{C} 1$ & & 111.1(2) & $\mathrm{N} 11 \mathrm{C} 2$ & $\mathrm{C} 22$ & & $.3(2)$ & C6 I & $3 \mathrm{~N} 8$ & $120.95(14)$ & $\mathrm{N} 2 \mathrm{~N} 4 \mathrm{C} 14$ & $112.1(4)$ \\
\hline N8 C10 C9 & & $108.5(2)$ & $\mathrm{N} 11 \mathrm{C} 2$ & $\mathrm{C} 47$ & & $.1(2)$ & N3 & $6 \mathrm{C} 19$ & $107.3(13)$ & N4 C6 C7 & $121.0(4)$ \\
\hline $\mathrm{C} 10 \mathrm{C} 9 \mathrm{C} 13$ & & $117.7(2)$ & $\mathrm{C} 44 \mathrm{C} 3$ & $\mathrm{C} 47$ & & $.3(2)$ & $\mathrm{C} 25$ & $\mathrm{C} 24 \mathrm{~N} 8$ & $116.8(2)$ & C7 C6 C13 & $119.6(4)$ \\
\hline $\mathrm{N} 8 \mathrm{C} 10 \mathrm{C} 1$ & & $110.6(2)$ & N8 C44 & 38 & & $.9(2)$ & $\mathrm{N} 3$ & $8 \mathrm{C} 24$ & $117.6(2)$ & $\mathrm{N} 2 \mathrm{C} 11 \mathrm{C} 18$ & $110.9(4)$ \\
\hline N3 N8 C10 & & $121.8(2)$ & $\mathrm{C} 20 \mathrm{C} 3$ & $\mathrm{C} 47$ & & $.3(2)$ & & & & $\mathrm{N} 2 \mathrm{C} 11 \mathrm{C} 20$ & $124.0(4)$ \\
\hline
\end{tabular}

\section{Experimental Section}

All melting points were measured on Gallenkamp electrothermal melting point apparatus and are uncorrected. IR spectra were recorded as $\mathrm{KBr}$ pellets on a Pye Unicam SP 3-300 Spectrophotometer. ${ }^{1} \mathrm{H}$ NMR spectra were recorded in deuterated dimethylsulfoxide (DMSO- $\mathrm{d}_{6}$ ) or deuterated chloroform $\left(\mathrm{CDCl}_{3}\right)$ at $\mathbf{3 0 0} \mathrm{MHz}$ on a Varian Gemini NMR spectrometer using tetramethylsilane (TMS) as an internal reference and results are expressed as $\delta$ values. Mass spectra were performed on a Shimadzu GCMS-QP 1000 Ex mass spectrometer at $70 \mathrm{eV}$. Elemental analyses were carried out at the Micro analytical center of Cairo University. Compounds 3a-j has been prepared as previously reported. ${ }^{8}$

\section{General procedure for preparation of enaminones 2a-d}

A mixture of heteroaryl ketones and methylaryl ketones 1a-d $(10 \mathrm{mmol})$ and dimethylformamide dimethylacetal DMFDMA $(10 \mathrm{mmol})$ with little excess, or in xylene was heated under reflux for 8 hours, and then left to cool at room temperature. The solid products 2a-d were collected by filtration, and then recrystallized form xylene.

3-Dimethylamino-1-(2-thienyl) propenone (2a), mp 122-124 ${ }^{\circ} \mathrm{C}\left(\mathrm{Lit}^{1} 132^{\circ} \mathrm{C}\right)$.

3-Dimethylamino-1-(2-pyridyl) propenone (2b), mp 135-136 ${ }^{\circ} \mathrm{C}\left(\mathrm{Lit}^{1} 134^{\circ} \mathrm{C}\right)$.

3-Dimethylamino-1- $\boldsymbol{p}$-tolyl propenone (2c), mp $94-96^{\circ} \mathrm{C}\left(\mathrm{Lit}^{14} 93-95{ }^{\circ} \mathrm{C}\right)$.

3-Dimethylamino-1-(4-nitrophenyl) propenone (2d), $\mathrm{mp} 152-154^{\circ} \mathrm{C}\left(\mathrm{Lit}^{14} 153^{\circ} \mathrm{C}\right)$. 
3-Dimethylamino-1-(4-chlorophenyl) propenone (2e), $\mathrm{mp} 86-88^{\circ} \mathrm{C}\left(\mathrm{Lit}^{14} 81-83{ }^{\circ} \mathrm{C}\right)$.

\section{General procedure for preparation of pyridazine derivatives $7 \mathbf{b}-\mathbf{f}, \mathbf{h}-\mathbf{j}$}

To a solution of $\mathrm{Ph}_{3} \mathrm{P}(10 \mathrm{mmol})$ and each of hydrazones $\mathbf{3 b - f}, \mathbf{h}-\mathbf{j}(10 \mathrm{mmol})$ in $\mathrm{CH}_{2} \mathrm{ClCH}_{2} \mathrm{Cl}$ $(10 \mathrm{ml})$ was added drop wise to a solution of dimethylacetylene dicarboxylate $(10 \mathrm{mmol})$. The mixture was left standing at room temperature for $24 \mathrm{hrs}$ and then treated with ethanol. The solid product was collected by filtration, then recrystallized from ethanol.

6-(Thiophene-2-carbonyl)-2-p-tolyl-2,3-dihydro-pyridazine-3,4-dicarboxylic acid dimethyl ester (7b). Orange crystals, yield (89\%); mp 184-185 $\mathrm{C}$. IR (KBr): v = 1676 (CO), 1651 (CO) $\mathrm{cm}^{-1} ;{ }^{1} \mathrm{H}$ NMR $\left(\mathrm{DMSO}_{6}\right): \delta=2.43\left(\mathrm{~s}, 3 \mathrm{H}, \mathrm{CH}_{3}\right), 3.66\left(\mathrm{~s}, 3 \mathrm{H}, \mathrm{OCH}_{3}\right), 3.86\left(\mathrm{~s}, 3 \mathrm{H}, \mathrm{OCH}_{3}\right), 6.26$ (s, 1H, HC-NAr) , 7.34-7.85(m, 7H, Ar-H), 7.81(s, 1H, H-5). MS (EI, 70eV): $m / z=398\left(\mathrm{M}^{+}\right)$. Anal. calcd for $\mathrm{C}_{20} \mathrm{H}_{18} \mathrm{~N}_{2} \mathrm{O}_{5} \mathrm{~S}$ (398.43): C, 60.29; H, 4.55; N, 7.03. Found: C, 60.30; H, 4.56; N, 7.20 .

2-(4-Bromophenyl)-6-(thiophene-2-carbonyl)-2,3-dihydropyridazine-3,4-dicarboxylic acid dimethyl ester (7c). Yellow crystals, yield (86\%); mp 172-174 ${ }^{\circ} \mathrm{C}$. IR (KBr): v = $1690(\mathrm{CO})$, $1620(\mathrm{CO}) \mathrm{cm}^{-1}$;- ${ }^{1} \mathrm{H}$ NMR $\left(\mathrm{DMSO}_{-} \mathrm{d}_{6}\right): \delta=3.66\left(\mathrm{~s}, 3 \mathrm{H}, \mathrm{OCH}_{3}\right), 3.86\left(\mathrm{~s}, 3 \mathrm{H}, \mathrm{OCH}_{3}\right), 6.26(\mathrm{~s}, 1 \mathrm{H}$, HC-NAr), 7.30-8.13 (m, 7H, Ar-H), 7.59 (s, 1H, H-5). - MS (EI, 70eV): $m / z=464\left(\mathrm{M}^{+}+1\right)$. Anal. calcd for $\mathrm{C}_{19} \mathrm{H}_{15} \mathrm{BrN}_{2} \mathrm{O}_{5} \mathrm{~S}$ (463.30): C, 49.26; H, 3.26; N, 6.05. Found: C, 49.31; H, 3.25; N, 6.22 .

2-(4-Methoxyphenyl)-6-(thiophene-2-carbonyl)-2,3-dihydro-pyridazine-3,4-dicarboxylic acid dimethyl ester (7d). Orange crystals, yield (85\%); mp 168-170 ${ }^{\circ} \mathrm{C}$, IR (KBr): v $=1695$ (CO), $1628(\mathrm{CO}) \mathrm{cm}^{-1} ;{ }^{1} \mathrm{H}$ NMR (DMSO-d $): \delta=3.68\left(\mathrm{~s}, 3 \mathrm{H}, \mathrm{OCH}_{3}\right), 3.84\left(\mathrm{~s}, 3 \mathrm{H}, \mathrm{OCH}_{3}\right), 3.90$ $\left(\mathrm{s}, 3 \mathrm{H}, \mathrm{OCH}_{3}\right), 6.26$ (s, 1H, HC-NAr), 6.99 (d, 2H, $\left.J=9 \mathrm{~Hz}, \mathrm{Ar}-\mathrm{H}\right), 7.09$ (d, 2H, J = $9 \mathrm{~Hz}$, Ar$\mathrm{H}), 7.63(\mathrm{~s}, 1 \mathrm{H}, \mathrm{H}-5), 7.71(\mathrm{~m}, 1 \mathrm{H}$, thiophene $\mathrm{H}-4), 8.01(\mathrm{~d}, 1 \mathrm{H}$, thiophene $\mathrm{H}-3), 8.40(\mathrm{~d}, 1 \mathrm{H}$, thiophene H-5); - MS (EI, 70eV): $m / z=414\left(\mathrm{M}^{+}\right)$. Anal. calcd for $\mathrm{C}_{20} \mathrm{H}_{18} \mathrm{~N}_{2} \mathrm{O}_{6} \mathrm{~S}$ (414.43): C, 57.96; H, 4.38; N, 6.76. Found: C, 58.00; H, 4.36; N, 6.83.

2-(4-Nitrophenyl)-6-(thiophene-2-carbonyl)-2,3-dihydro-pyridazine-3,4-dicarboxylic acid dimethyl ester (7e). Orange crystals, yield (90\%); mp 286-288 ${ }^{\circ} \mathrm{C}, \mathrm{IR}(\mathrm{KBr}): \mathrm{v}=1708(\mathrm{CO})$, $1635(\mathrm{CO}) \mathrm{cm}^{-1} ;{ }^{1} \mathrm{H}$ NMR $\left(\mathrm{DMSO}_{6}\right): \delta=3.65\left(\mathrm{~s}, 3 \mathrm{H}, \mathrm{OCH}_{3}\right), 3.87\left(\mathrm{~s}, 3 \mathrm{H}, \mathrm{OCH}_{3}\right) 6.38(\mathrm{~s}, 1 \mathrm{H}$, HC-NAr), 7.29-7.32 (m, 1H, thiophene H-4), 7.71 (s, 1H, H-5), 7.90 (d, 2H, J = 9 Hz, Ar-H), 8.10-8.14 (m, 2H, thiophene H-3,5), $8.35(\mathrm{~d}, 2 \mathrm{H}, J=9 \mathrm{~Hz}, \mathrm{Ar}-\mathrm{H}) ;{ }^{13} \mathrm{C}$ NMR (DMSO- $\left.\mathrm{d}_{6}\right): \delta=$ $52.76\left(\mathrm{CH}_{3}\right), 53.30\left(\mathrm{CH}_{3}\right), 88.50(\mathrm{C}-3), 118.04\left(\mathrm{C}-2^{\prime}, 6^{\prime}, \mathrm{Ar}\right), 125.22$ (C-3`, 5', Ar), $128.95(\mathrm{C}-4$, thiophene), 133.20 (C-5, thiophene), 135.64 (C-3, thiophene), 136.88 (C-4', Ar), 143.56 (C-2, thiophene), 144.60 (C-4), 146.05 (C-1', Ar), 149.20 (C-5), 162.56 (CO), 168.90 (CO), 175.60 (CO). - MS (EI, 70eV): $m / z=429\left(\mathrm{M}^{+}\right)$. Anal. calcd for $\mathrm{C}_{19} \mathrm{H}_{15} \mathrm{~N}_{3} \mathrm{O}_{7} \mathrm{~S}$ (429.40): C, 53.14; $\mathrm{H}$, $3.52 ; \mathrm{N}, 9.79$. Found: C, 53.18; H, 3.50; N, 9.82 .

6-(4-Methylbenzoyl)-2-phenyl-2,3-dihydro-pyridazine-3,4-dicarboxylic acid dimethyl ester (7f). Orange crystals, yield (81\%); mp 156-157 ${ }^{\circ} \mathrm{C}$; IR (KBr): v = $1700(\mathrm{CO}), 1624(\mathrm{CO}) \mathrm{cm}^{-1}$;${ }^{1} \mathrm{H}$ NMR $\left(\mathrm{DMSO}-\mathrm{d}_{6}\right): \delta=2.39\left(\mathrm{~s}, 3 \mathrm{H}, \mathrm{CH}_{3}\right), 3.67\left(\mathrm{~s}, 3 \mathrm{H}, \mathrm{OCH}_{3}\right), 3.85\left(\mathrm{~s}, 3 \mathrm{H}, \mathrm{OCH}_{3}\right), 6.25(\mathrm{~s}$, 1H, HC-NAr), 7.22-7.89 (m, 9H, Ar-H) and 7.60 (s, 1H, H-5). - MS (EI, 70eV): $\mathrm{m} / z=333\left(\mathrm{M}^{+}-\right.$ 
59). Anal. calcd for $\mathrm{C}_{22} \mathrm{H}_{20} \mathrm{~N}_{2} \mathrm{O}_{5}$ (392.40): C, 67.34; H, 5.14; N, 7.14. Found: C, 67.70; H, 5.20; N, 7.22.

2-(4-Bromophenyl)-6-(4-methylbenzoyl)-2,3-dihydro-pyridazine-3,4-dicarboxylic acid dimethyl ester (7h). Orange crystals, yield (82\%); mp 201-203 ${ }^{\circ} \mathrm{C}$; IR (KBr): v = 1699 (CO), $1629(\mathrm{CO}) \mathrm{cm}^{-1}$;- ${ }^{1} \mathrm{H}$ NMR (DMSO-d 6 ): $\delta=2.41\left(\mathrm{~s}, 3 \mathrm{H}, \mathrm{CH}_{3}\right), 3.67\left(\mathrm{~s}, 3 \mathrm{H}, \mathrm{OCH}_{3}\right), 3.86(\mathrm{~s}, 3 \mathrm{H}$, $\left.\mathrm{OCH}_{3}\right), 6.26$ (s, 1H, HC-NAr), 7.34-7.85 (m, 8H, Ar-H), 7.81(s, 1H, H-5). - MS (EI, 70eV): $\mathrm{m} / z$ $=413\left(\mathrm{M}^{+}\right.$-58). Anal. calcd for $\mathrm{C}_{22} \mathrm{H}_{19} \mathrm{BrN}_{2} \mathrm{O}_{5}$ (471.30): C, 56.07; H, 4.06; N, 5.94. Found: C, $56.20 ; \mathrm{H}, 4.00 ; \mathrm{N}, 6.00$.

2-(4-Methoxyphenyl)-6-(4-methylbenzoyl)-2,3-dihydro-pyridazine-3,4-dicarboxylic acid dimethyl ester (7i). Orange crystals, yield (79\%); mp 212-214 ${ }^{\circ} \mathrm{C}$; IR (KBr): v = $1690(\mathrm{CO})$, $1635(\mathrm{CO}) \mathrm{cm}^{-1} ;{ }^{1} \mathrm{H}$ NMR $\left(\mathrm{DMSO}_{-} \mathrm{d}_{6}\right): \delta=2.39\left(\mathrm{~s}, 3 \mathrm{H}, \mathrm{CH}_{3}\right), 3.65\left(\mathrm{~s}, 3 \mathrm{H}, \mathrm{OCH}_{3}\right), 3.76(\mathrm{~s}, 3 \mathrm{H}$, $\left.\mathrm{OCH}_{3}\right), 3.86\left(\mathrm{~s}, 3 \mathrm{H}, \mathrm{OCH}_{3}\right), 6.24(\mathrm{~s}, 1 \mathrm{H}, \mathrm{HC}-\mathrm{NAr}), 6.96(\mathrm{~d}, 2 \mathrm{H}, J=8.4 \mathrm{~Hz}, \mathrm{Ar}-\mathrm{H}), 7.22$ (d, 2H, $J$ = 8.4 Hz, Ar-H), 7.27 (d, 2H, $J=9 \mathrm{~Hz}, \mathrm{Ar}-\mathrm{H}), 7.59$ (s, 1H, H-5), 7.85 (d, 2H, J=9 Hz, Ar-H), MS (EI, 70eV): $m / z=422\left(\mathrm{M}^{+}\right)$. Anal. calcd for $\mathrm{C}_{23} \mathrm{H}_{22} \mathrm{~N}_{2} \mathrm{O}_{6}$ (422.43): C, 65.39; H, 5.25; N, 6.63. Found: C, 65.45; H, 5.32; N, 6.45 .

2-(4-Bromophenyl)-6-(4-nitrobenzoyl)-2,3-dihydro-pyridazine-3,4-dicarboxylic acid dimethyl ester (7j). Yellow crystals, yield (91\%); mp 204-206 ${ }^{\circ} \mathrm{C}$; IR (KBr): v = 1705 (CO), $1638(\mathrm{CO}) \mathrm{cm}^{-1}$; ${ }^{1} \mathrm{H}$ NMR; (DMSO-d $): 3.78\left(\mathrm{~s}, 3 \mathrm{H}, \mathrm{OCH}_{3}\right), 3.86\left(\mathrm{~s}, 3 \mathrm{H}, \mathrm{OCH}_{3}\right), 6.25(\mathrm{~s}, 1 \mathrm{H}$, HC-NAr), 7.04-8.75 (m, 8H, Ar-H), 7.07 (s, 1H, H-5). MS; m/z $503\left(\mathrm{M}^{+}+1\right)$. Anal. calcd for $\mathrm{C}_{21} \mathrm{H}_{16} \mathrm{BrN}_{3} \mathrm{O}_{7}$ (502.27): C, 50.22; H, 3.21; N, 8.37. Found: C, 50.23; H, 3.22; N, 8.24.

\section{General procedure for preparation of pyrazole derivatives $9 \mathrm{~b}, \mathrm{~d}, \mathbf{f}, \mathrm{g}$}

Each of 2-arylazopropanals $\mathbf{3 b}, \mathbf{d}, \mathbf{f}, \mathbf{g}(10 \mathrm{mmol}), \mathrm{PhCOCH}_{2} \mathrm{Br}(10 \mathrm{mmol})$ and potassium carbonate anhydrous $(10 \mathrm{mmol})$ in acetone was heated under reflux for $6 \mathrm{hrs}$ in water bath. The reaction mixture was filtered, and then left to cool at room temperature. The solid product was collected by filtration and crystallized from ethanol.

(5-Benzoyl-1-p-tolyl-1H-pyrazol-3-yl)-thiophen-2-yl-methanone (9b). Orange crystals, yield (88\%); mp 206-208 ${ }^{\circ} \mathrm{C}$; IR (KBr): $v=1680$ (CO), 1648 (CO) $\mathrm{cm}^{-1} ;{ }^{1} \mathrm{H}$ NMR (DMSO-d $): \delta=$ $2.37\left(\mathrm{~s}, 3 \mathrm{H}, \mathrm{CH}_{3}\right), 7.29-7.32(\mathrm{~m}, 3 \mathrm{H}, \mathrm{Ar}-\mathrm{H}), 7.38(\mathrm{~s}, 1 \mathrm{H}$, pyrazole $\mathrm{H}-4), 7.44(\mathrm{~d}, 2 \mathrm{H}, J=8.5 \mathrm{~Hz}$, Ar-H), 7.59 (t, 2H, $J=7.8 \mathrm{~Hz}, \mathrm{Ar}-\mathrm{H}), 7.71-7.77$ (m, 1H, thiophene H-4), 7.94 (d, 2H, $J=8.5 \mathrm{~Hz}$, $\operatorname{Ar}-\mathrm{H}), 8.11(\mathrm{~d}, 1 \mathrm{H}$, thiophene $\mathrm{H}-3), 8.48(\mathrm{~d}, 1 \mathrm{H}$, thiophene $\mathrm{H}-5) ;{ }^{13} \mathrm{C}$ NMR $\left(\mathrm{DMSO}-\mathrm{d}_{6}\right): \delta=$ $20.59\left(\mathrm{CH}_{3}\right), 114.21(\mathrm{C}-4), 120.81$ (C-5), 124.62, 128.25, 128.86, 129.53, 129.65 (C-4, thiophene), 134.17, 136.04 (C-3, thiophene), 136.25, 136.96, 138.54 (C-5, thiophene), 140.26, 141.32 (C-2, thiophene), 149.21 (C-3), 177.47 (CO), 184.82 (CO). MS (EI, 70eV): $\mathrm{m} / z=372$ $\left(\mathrm{M}^{+}\right)$. Anal. calcd for $\mathrm{C}_{22} \mathrm{H}_{16} \mathrm{~N}_{2} \mathrm{O}_{2} \mathrm{~S}$ (372.44): C, 70.95; H, 4.33; N, 7.52. Found: C, 71.00; H, $4.35 ; \mathrm{N}, 7.55$.

]5-Benzoyl-1-(4-methoxyphenyl)-1H-pyrazol-3-yl[-thiophen-2-yl-methanone (9d). Orange crystals, yield (81\%); mp 237-239 $\mathrm{C}$; IR (KBr): $v=1671(\mathrm{CO}), 1639$ (CO) cm ${ }^{-1}$; ${ }^{1} \mathrm{H}$ NMR $\left(\mathrm{DMSO}_{-} \mathrm{d}_{6}\right): \delta=3.71\left(\mathrm{~s}, 3 \mathrm{H}, \mathrm{OCH}_{3}\right), 6.91(\mathrm{~s}, 1 \mathrm{H}, \mathrm{H}-4$ pyrazole), 7.57-8.21 (m, 12H, 9 Ar-H and 
3H-thienyl). - MS (EI, 70eV): $m / z=388\left(\mathrm{M}^{+}\right)$. Anal. calcd for $\mathrm{C}_{22} \mathrm{H}_{16} \mathrm{~N}_{2} \mathrm{O}_{3} \mathrm{~S}$ (388.44): C, 68.02; H, 4.15; N, 7.21. Found: C68.21; H, 4.16; N, 7.33

(5-Benzoyl-1-phenyl-1H-pyrazol-3-yl)-p-tolylmethanone (9f). Yellow crystals, yield (90\%); mp 158-159 $\mathrm{C}$; IR (KBr): $v=1675$ (CO), 1625 (CO) $\mathrm{cm}^{-1}$;- ${ }^{1} \mathrm{H}$ NMR (DMSO-d 6 ): $\delta=2.40(\mathrm{~s}$, $3 \mathrm{H}, \mathrm{CH}_{3}$ ), 7.36 (s, 1H, H-4 pyrazole), 7.39-8.19 (m, 14H, Ar-H). MS (EI, 70eV): $m / z=366$ $\left(\mathrm{M}^{+}\right)$. Anal. calcd for $\mathrm{C}_{24} \mathrm{H}_{18} \mathrm{~N}_{2} \mathrm{O}_{2}$ (366.41): C, 78.67; H, 4.95; N, 7.65 Found: C, 78.60; H, 5.00; N, 7.50.

(5-Benzoyl-1-p-tolyl-1H-pyrazol-3-yl)-p-tolylmethanone(9g). Orange crystals, yield (89\%); mp 164-166 ${ }^{\circ}$; IR (KBr): $v=1679$ (CO), $1629(\mathrm{CO}) \mathrm{cm}^{-1}$;- ${ }^{1} \mathrm{H}$ NMR (DMSO-d $\left.{ }_{6}\right): \delta=2.36(\mathrm{~s}$, $\left.3 \mathrm{H}, \mathrm{CH}_{3}\right), 2.41$ (s, 3H, $\left.\mathrm{CH}_{3}\right), 7.28$ (s, 1H, H-4 pyrazole), 7.30-8.19 (m, 13H, Ar-H). - MS (EI, $70 \mathrm{eV}): m / z=380\left(\mathrm{M}^{+}\right)$. Anal. calcd for $\mathrm{C}_{25} \mathrm{H}_{20} \mathrm{~N}_{2} \mathrm{O}_{2}$ (380.44): C, 78.93; H, 5.30; N, 7.36. Found: C, 78.81; H, 5.47; N, 7.12.

\section{5-(Benzoyl-6-imino-1- p-tolyl-1,6-dihydropyridazin-3-yl)-p-tolyl-methanone (11).}

To a solution of $\mathbf{3 g}(10 \mathrm{mmol})$ and benzoylacetonitrile $(10 \mathrm{mmol})$ in ethanol $(50 \mathrm{ml})$ were added a few drops of piperidine. The reaction mixture was heated under reflux for 2 hours. Then the solvent was evaporated and the solid so formed, was collected by filtration and then recrystallized from ethanol. Orange crystals, yield (89\%); mp 213-215 ${ }^{\circ} \mathrm{C}$; IR ( $\left.\mathrm{KBr}\right): v=3244$ $(\mathrm{NH}), 1660(\mathrm{CO}), 1625(\mathrm{CO}) \mathrm{cm}^{-1} ;{ }^{1} \mathrm{H}$ NMR $\left(\mathrm{DMSO}_{-} \mathrm{d}_{6}\right): \delta=2.36\left(\mathrm{~s}, 3 \mathrm{H}, \mathrm{CH}_{3}\right), 2.44(\mathrm{~s}, 3 \mathrm{H}$, $\left.\mathrm{CH}_{3}\right), 7.22-8.01\left(\mathrm{~m}, 14 \mathrm{H}, \mathrm{Ar}-\mathrm{H}, \mathrm{NH}\right.$ and $4 \mathrm{H}$ pyridazine). - MS (EI, 70eV): $\mathrm{m} / z=407\left(\mathrm{M}^{+}\right)$. Anal. calcd for $\mathrm{C}_{26} \mathrm{H}_{21} \mathrm{~N}_{3} \mathrm{O}_{2}$ (407.46): C, 76.64; H, 5.19; N, 10.31. Found: C, 76.60; H, 5.25; N, 10.44.

\section{General method for preparation of $12 \mathrm{c}-\mathrm{e}, \mathrm{h}, \mathrm{j}$ via reaction with $p$-toluidine}

A mixture of compound 3c-e,h,j $(10 \mathrm{mmol})$ and $p$-toluidine $(10 \mathrm{mmol}, 1.07 \mathrm{~g})$ in ethanol was refluxed for 3 hours. The solvent was evaporated and the solid so formed, was collected by filtration and then recrystallized from ethanol.

2-(4-Bromophenylazo)-1-thiophen-2-yl-3-p-tolylaminopropenone (12c). Orange crystals, yield (34\%), mp. 197-199 C. IR (KBr): v=3258(NH), $1626(\mathrm{CO}) \mathrm{cm}^{-1}$; ${ }^{1} \mathrm{H}$ NMR (DMSO-d 6 ): $\delta=2.35\left(\mathrm{~s}, 3 \mathrm{H}, \mathrm{CH}_{3}\right), 7.25-8.09(\mathrm{~m}, 11 \mathrm{H}, \mathrm{Ar}-\mathrm{H}), 8.76(\mathrm{~d}, 1 \mathrm{H}, J=6.9 \mathrm{~Hz}, H \mathrm{C}-\mathrm{NH}), 15.14(\mathrm{~d}, 1 \mathrm{H}$, $J=6.9 \mathrm{~Hz}, \mathrm{NH})$. MS (EI, 70eV): $m / z=425\left(\mathrm{M}^{+}-1\right)$. - Anal. calcd for $\mathrm{C}_{20} \mathrm{H}_{16} \mathrm{BrN}_{3} \mathrm{OS}$ (426.33): C 56.34, H 3.78, N 9.86. Found C 56.33, H 3.79, N 9.65.

2-(4-Methoxyphenylazo)-1-thiophen-2-yl-3-p-tolylaminopropenone (12d). Orange crystals, yield (40\%), mp. $164-166^{\circ} \mathrm{C}$; IR (KBr): $v=3263(\mathrm{NH}), 1651(\mathrm{CO}) \mathrm{cm}^{-1}$; ${ }^{1} \mathrm{H}$ NMR (DMSO-d 6 ): $\delta=2.34\left(\mathrm{~s}, 3 \mathrm{H}, \mathrm{CH}_{3}\right), 3.75\left(\mathrm{~s}, 3 \mathrm{H}, \mathrm{OCH}_{3}\right), 7.08-8.08(\mathrm{~m}, 11 \mathrm{H}, \mathrm{Ar}-\mathrm{H}), 8.63(\mathrm{~d}, 1 \mathrm{H}, J=7.2 \mathrm{~Hz}$, $H \mathrm{C}-\mathrm{NH}), 14.73(\mathrm{~d}, 1 \mathrm{H}, J=7.2 \mathrm{~Hz}, \mathrm{NH})-\mathrm{MS}(\mathrm{EI}, 70 \mathrm{eV}): m / z=377\left(\mathrm{M}^{+}\right)$. Anal. calcd for $\mathrm{C}_{21} \mathrm{H}_{19} \mathrm{~N}_{3} \mathrm{O}_{2} \mathrm{~S}$ (377.46): C 66.82, H 5.07, N 11.13. Found C 66.59, H 5.06, N 11.27.

2-(4-Nitrophenylazo)-1-thiophen-2-yl-3-p-tolylaminopropenone (12e). Orange crystals, yield (43\%), mp. $225-227^{\circ} \mathrm{C}$; IR (KBr): $v=3297(\mathrm{NH}), 1652(\mathrm{CO}) \mathrm{cm}^{-1} ;{ }^{1} \mathrm{H}$ NMR (DMSO-d 6 ): $\delta=$ $2.38\left(\mathrm{~s}, 3 \mathrm{H}, \mathrm{CH}_{3}\right), 7.31-8.37(\mathrm{~m}, 11 \mathrm{H}, \mathrm{Ar}-\mathrm{H}), 8.90(\mathrm{~d}, J=6.9 \mathrm{~Hz}, 1 \mathrm{H}, H \mathrm{C}-\mathrm{NH}), 15.56(\mathrm{~d}, J=6.9$ $\mathrm{Hz}, 1 \mathrm{H}, \mathrm{NH})-\mathrm{MS}(\mathrm{EI}, 70 \mathrm{eV}): m / z=392\left(\mathrm{M}^{+}\right)$. Anal. calcd for $\mathrm{C}_{20} \mathrm{H}_{16} \mathrm{~N}_{4} \mathrm{O}_{3} \mathrm{~S}$ (392.43): C 61.21, H 4.11, N 14.28. Found C 61.34, H 4.10, N 14.33. 
2-(4-Bromophenylazo)-1-p-tolyl-3-p-tolylaminopropenone (12h). Orange crystals, yield $(66 \%), \mathrm{mp} .166-168^{\circ} \mathrm{C}$. IR $(\mathrm{KBr}): \mathrm{v}=3417(\mathrm{NH}), 1635(\mathrm{CO}) \mathrm{cm}^{-1} ;{ }^{1} \mathrm{H}$ NMR (DMSO-d 6$): \delta=$ $2.35\left(\mathrm{~s}, 3 \mathrm{H}, \mathrm{CH}_{3}\right), 2,41\left(\mathrm{~s}, 3 \mathrm{H}, \mathrm{CH}_{3}\right), 7.28-8.30(\mathrm{~m}, 12 \mathrm{H}, \mathrm{Ar}-\mathrm{H}), 8.79$ (d , $\left.J=6.7 \mathrm{~Hz}, 1 \mathrm{H}, H \mathrm{C}-\mathrm{NH}\right)$, $15.14(\mathrm{~d}, 1 \mathrm{H}, J=6.7 \mathrm{~Hz}, \mathrm{NH})$ - MS (EI, 70eV): $\mathrm{m} / z=434\left(\mathrm{M}^{+}\right)$. - Anal. calcd for $\mathrm{C}_{23} \mathrm{H}_{20} \mathrm{BrN}_{3} \mathrm{O}$ (434.33): C 63.60, H 4.64, N 9.67. Found C 63.61, H 4.62, N 9.49.

2-(4-Bromophenylazo)-1-(4-nitrophenyl)-3-p-tolylaminopropenone (12j). Orange crystals, yield $(40 \%)$, mp. $234-236^{\circ} \mathrm{C}$. IR (KBr): $v=3417(\mathrm{NH}), 1643(\mathrm{CO}) \mathrm{cm}^{-1} ;{ }^{1} \mathrm{H}$ NMR (DMSO-d $): \delta$ $=2.36\left(\mathrm{~s}, 3 \mathrm{H}, \mathrm{CH}_{3}\right), 7.27(\mathrm{~d}, 2 \mathrm{H}, J=8.1 \mathrm{~Hz}, \mathrm{Ar}-\mathrm{H}), 7.44(\mathrm{~d}, 2 \mathrm{H}, J=8.1 \mathrm{~Hz}, \mathrm{Ar}-\mathrm{H}), 7.52(\mathrm{~d}, 2 \mathrm{H}$, $J=8.4 \mathrm{~Hz}, \mathrm{Ar}-\mathrm{H}), 7.69$ (d, 2H, $J=8.4 \mathrm{~Hz}, \mathrm{Ar}-\mathrm{H}), 8.05$ (d, 2H, $J=8.7 \mathrm{~Hz}, \operatorname{Ar}-\mathrm{H}), 8.36$ (d, 2H, $J$ $=8.7 \mathrm{~Hz}, \mathrm{Ar}-\mathrm{H}), 8.77$ (d , $J=7 \mathrm{~Hz}, 1 \mathrm{H}, H \mathrm{C}-\mathrm{NH}), 14.94$ (d, 1H, $J=7 \mathrm{~Hz}, \mathrm{NH})-\mathrm{MS}$ (EI, 70eV): $m / z=464\left(\mathrm{M}^{+}-1\right)$. - Anal. calcd for $\mathrm{C}_{22} \mathrm{H}_{17} \mathrm{BrN}_{4} \mathrm{O}_{3}$ (465.30): C 56.79, H 3.68, N 12.04. Found C 56.88, H 3.67, N 12.30.

\section{References}

1. Al-Omran, F.; Abdel-Khalik, M. M.; El-Khair, A. A.; Elnagdi, M. H. Synthesis 1997, 91.

2. Ghozlan, S. A .S; Abdelhamid, I. A.; Elnagdi, M. H. Arkivoc 2006, 147.

3. Al-Awadi, N. A.; Elnagdi, M. H.; Ibrahim, Y. A.; Kaul, K.; Kumar, A. Tetrahedron 2001, 57, 1609.

4. Al-Zaydi, K. M.; Hafez, E. A.; Elnagdi, M. H. J. Chem. Res. (S), 2000, 154; (M) 2000, 510.

5. Al-Shiekh, M. A.; Salaheldin, A. M.; Hafez, E. A.; Elnagdi, M. H. J. Chem. Res. 2004, 3, 174.

6. Al-Shiekh, M. A.; Salaheldin, A. M.; Hafez, E. A.; Elnagdi, M. H. J. Heterocyclic Chem. 2004, 41, 647.

7. Tseng, S.; Esptein, J. W.; Brbaander, H. J.; Francisco, G. J. Heterocyclic Chem. 1987, 24, 837.

8. Al-Shiekh, M. A.; Medrassi, H. Y.; Elnagdi, M. H.; Hafez, E. A. J. Chem. Res. 2007, 432.

9. Kenawi,I. M.; Elnagdi, M. H. Spectrochim. Acta (A) 2006, 65, 805.

10. CCDC $617786,617787,617788$ and 617777 contain the supplementary crystallographic data for this paper. These data can be obtained free of charge from The Cambridge Crystallographic Data Center via. http://www.ccdc.ac.uk/data request/cif.

11. Al-Saleh, B.; El-Apassery, M. A.; Elnagdi, M. H. J. Chem. Res.. 2004, 578.

12. Altomare, A.; Cascarano, G.; Giacorazzo, C.; Guaghardi, A.; Burla, M. C.; Polidori, G.; Camalli, M. J. Appl. Cryst. 1994, 27, 435.

13. Johnson, C. K. ORTEP-11, Oak Ridge National Laboratory, Tennessee, USA, 1976.

14. Almazroa, S.; Elnagdi, M. H.; Salaheldin, A. M. J. Heterocyclic Chem. 2004, 41, 267. 